\title{
The particularities of near-critical flows formation in open channels
}

\author{
Oleksandr Riabenko ${ }^{1}$, Oksana Kliukha ${ }^{1}$, Volodymyr Tumoshchuk ${ }^{1}$, and Oksana Halych ${ }^{1, *}$ \\ ${ }^{1}$ National University of Water and Environmental Engineering, Rivne, Soborna St. 11, 33028, \\ Ukraine
}

\begin{abstract}
This article considers the current problems of near-critical flows. It provides short characteristics of each phenomenon and describes the cases of near-critical flow formation during the operation of the different hydraulic structures. Each of the considered phenomena has a number of characteristic features which distinguish them from the usual subcritical and supercritical flows with smooth or slowly varied movement. Such properties include the wave-like or roller nature of free-surface curves, the presence of a streamline inclination and curvature, and also a non-hydrostatic pressure distribution in depth mainly in the vertical cross-section of these phenomena. Therefore, during mathematical and numerical simulations at the designing stage of hydraulic structures it is necessary to take into account the additional parameters which characterise the particularities of near-critical flows. In cases in which these moments are neglected, there are many cases of accidents and damage being caused to structures which are operated in conditions of near-critical flow formation. An objective of this work is to provide a detailed analysis of the particularities of near-critical flows and show their negative consequences on hydraulic structures. The article presents the results of the mathematical and hydraulic simulation of wavelike near-critical flows and a comparison of the full-scale measurement and a mathematical model of translation waves.
\end{abstract}

\section{Introduction}

Current issues of water resources management are often connected with the production of electricity for fulfilling the energy needs of society [1] and providing flood control. The solution of these problems requires the building of different types of hydraulic constructions, such as water spillways, sluice gates, regulators, dams, dikes, and channels. The practice of operating such types of construction shows the possibility of the occurrence of not only subcritical and supercritical flows but also near-critical flows. Near-critical flows are freesurface water flows that are steady with depths, which are close to a critical value, and also unsteady flow (translational waves) with rapidly varying movement and velocities, which are close to a critical value. The domain of these flows includes different hydraulic phenomena, the most notable of which are solitary wave, cnoidal waves, undular jump, translation waves [2].

\footnotetext{
* Corresponding author: o.halych@gmail.com
} 
Near-critical flows have a number of characteristic properties which appreciably distinguish these flows from usual subcritical and supercritical flows of smooth or slowly varied movement. Such properties include the wave-like or roller nature of free-surface curves, the availability of inclination and curvature, non-hydrostatic pressure distribution in the vertical cross-section of these phenomena, the height of the phenomena, the bottom roller with negative velocity, and non-uniform pressure on the banks and bottom of channels due to the wave-like free-surface of phenomena, the large length of wave attenuation, etc.

The aforementioned properties can have a negative influence on the bank and bottom protection of channels, can cause different types of damage to hydraulic structures and can sometimes lead to the destruction of structures. Attempts to avoid the formation of these flows in open channels or in the operation of hydraulic structures are not always successful and they require significant additional funds; sometimes it is impossible to avoid the formation of such flows and therefore, the particularities of near-critical flows should be taken into account during the design, construction and operation of the hydraulic structures. For instance, the propagation of downstream free-surface waves must be taken into account for the design of canals and for the maintenance of natural channels due to the fact that wave height is an important design parameter that determines the required height of the canal sidewalls. In natural channels, the embankment height must be higher that the crest of the free-surface undulations to prevent overtopping and the subsequent erosion ultimately leading to the destruction of the banks. Furthermore, the propagation of free-surface waves might impose additional impact loads, perturbations and vibrations on downstream canal structures, etc. [3-4].

\section{Cases of near-critical occurrence}

According to regulatory instrument the occurrence of near-critical flows during operating various types of hydraulic structures must be prevented. However, the experience of operating hydraulic structures shows that during operation of the different types of hydraulic constructers, near-critical flows can occur (Fig. 1) [2].

\subsection{The occurrence of near-critical flows in the tail water of low head water spillways}

Analysis of the existed information on the operation of different types of spillways shows many cases of near-critical flows formation in the tail water of these structures, i.e. the roundcrested weir with the smooth conjugacy of the discharge surface and the downstream apron or baffle sill, behind broad-crested weir and sluice gates [3]. The near-critical flow formation is observed on flood controlling structures such as regulators, which are used for depth and flow regulating in channels, during flood routing, the management of hydraulic regimes, the protection of the riverbank from erosion, etc. The experimental studying of these flows during the operation of structures shows the low intensity of energy dissipation and wavelike velocity distribution along the channels - the maximum values of velocity occur under troughs and the minimum occur under crests of undular flows. Under some conditions, the considered phenomena occur in the tail water of run-of-river hydropower plants or in the tailrace channels of small-scale hydropower plants.

The above-mentioned features of spillway operating are significant in cases when they are used as measured structures and near-critical flow formation can lead to miscalculation during the prediction of the obtained measured results [3]. 

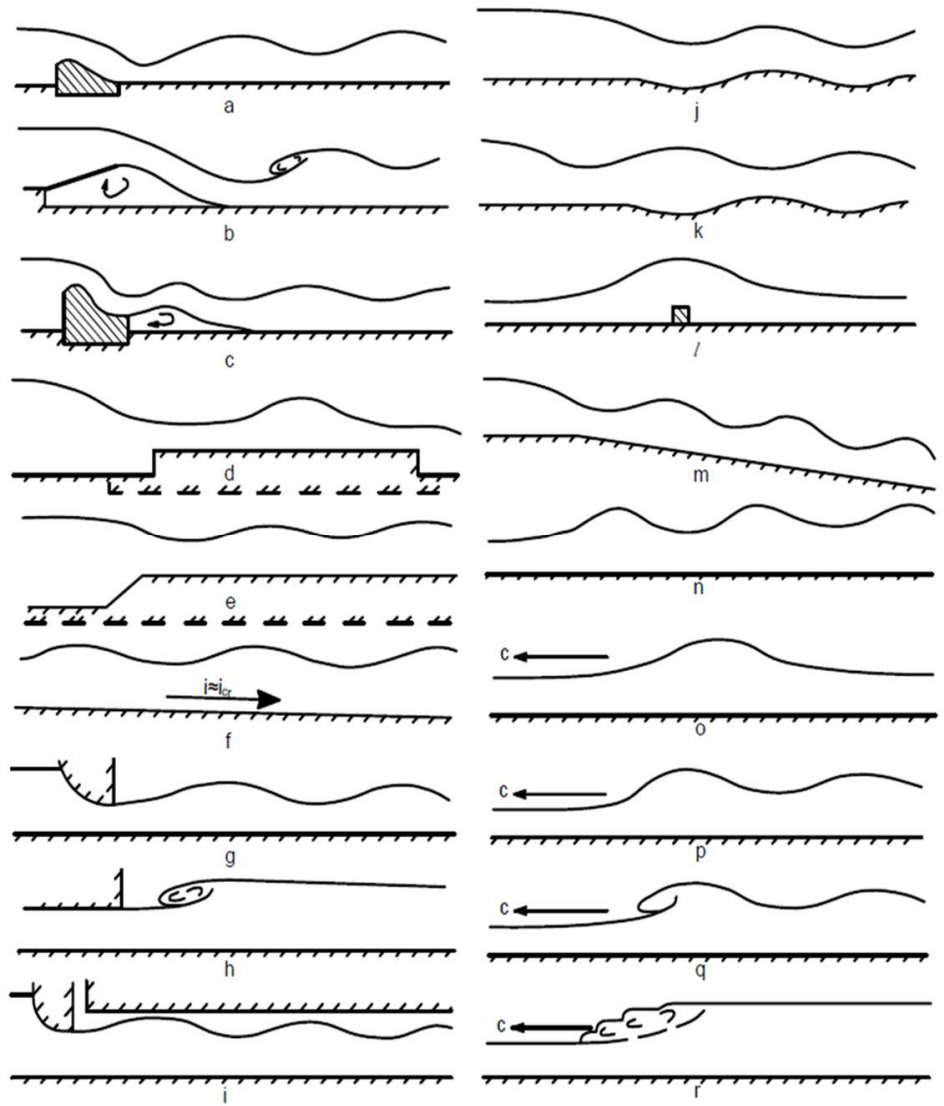

Fig. 1. Cases of near-critical flows formation: $a$ - behind spillways; $b$ - behind regulator sluices and low pressure control structures; $\mathrm{c}$ - behind spillways with drop wall and combined hydropower plants; $\mathrm{d}$ - on broad-crested weirs; $\mathrm{e}$ - at the inlet of channels or free-flow tunnels; $\mathrm{f}$ - in open channels, freeflow tunnels and pipes; $\mathrm{g}$ - during outflow from under gates with round or acicular configuration of bottom part; $h$ - at the outlet of bottom discharges; $i$ - in tunnels with boom; $\mathrm{j}, \mathrm{k}$ - over undular bottom; 1 - during flow-around of bottom obstacle; $\mathrm{m}$ - at the inlet of inclined drops; $\mathrm{n}$ - during controlling of supercritical flows; $\mathrm{o}, \mathrm{p}, \mathrm{q}, \mathrm{r}-$ in the form of translational waves.

\subsection{The occurrence of near-critical flows in free-flow tunnels, pipes and galleries}

One of the reasons for damage and flaws in free-flow tunnels, pipes and galleries is the occurrence of adverse hydraulic regimes that include near-critical flows. The difficulty of observing the wave processes which can occur inside the tunnels, pipes and galleries during their operation makes hydraulic simulation an integral part of their design.

There have been cases of changing the configuration or parts of tunnels after investigating the possibility of the formation of near-critical flows to avoid them. Based on laboratory investigation, the slope and configuration of the intake part of tunnel of the Enguri dam was changed to reduce the wave heights. A unique construction of an energy dissipator was designed following the discovery of immobile steady waves in the free-flow tailrace tunnel of the San Rock hydraulic complex [4]. 


\subsection{The occurrence of steady and unsteady near-critical flows in channels, rivers, water reservoirs and seas}

Lots of full-scale studying and laboratory experiments show that in channels, rivers, water reservoirs and seas etc., immobile and mobile near-critical flows with undular free-surface can form under some conditions. In some cases, the maximum height of the considered flows can be more than $80-100 \%$ of the average height of these waves [6]. This height influences the level of the top of the side walls and the shelf edge of channels, the bottom of the bridge beams, the height of the protective dikes, the hydraulic tunnels, pipes, galleries, and the diameter of the pipelines, etc.

The negative influence of near-critical flows caused the destruction of the bank and bottom concrete paving of the Karshynskyi, Amu-Bukharskyi and Sherabadskyi canals. Moreover, because of exceeding the wave crests over the calculated average water level, there was a flooding berm of the Karshynskyi canals along a length of 3.6-3.9 km [3].

Immobile wave-like near-critical flows can occur at the entrance of long channels and also in channels with slopes close to the critical value.

The mobile types of near-critical flows include translation waves and tidal bores. Translation waves occur during: the manoeuvring of gates of hydraulic structures; the destruction of dams; the startup, operation, capacity regulation and stopping of the hydraulic units of pumped storage power plants (PSPP), hydropower plants (HPP) and pumped stations (PS) in the forebay, upper water reservoir and tailrace channels [7]. Such waves form in the upper water reservoir of Dniester PSPP, in the headrace channel of Sharabadska PS, in the diversion channel of Orezon HPP, etc.

Tidal bores occur due to the action of tides in downstream flows of rivers which run into the sea or ocean.

\subsection{The occurrence of near-critical flows during supercritical flow management}

During the design, construction and operation of water spillways, supplies, pound locks and other types of free-flow hydraulic structures there are quite often issues related to the management of supercritical flows. During the operation of hydraulic structures such as: high-velocity channels, free-flow tunnels and water spillways, oblique wave-like near-critical flows might occur at the entrance of structures, spread along a large length and reach large heights. The formation of such waves causes the occurrence of impulsive pressure on the sidewalls of high-velocity channels and tunnels, the overflow of water from the edge of channels, etc.

In such a condition, the aim of supercritical flow management is the avoidance of such hydraulic regimes or decreasing the heights of oblique waves [3].

\section{Simulation of near-critical flows}

\subsection{Hydraulic and mathematical simulation of undular jump}

As mentioned above, to estimate the influence of near-critical flow parameters on the operation of hydraulic structures, it is recommended to use mathematical and hydraulic simulation. During the theoretical studying of near-critical flows a mathematical model was developed to calculate the free-surface profile of undular jump and cnoidal waves that is based on the possible presence of non-hydrostatic pressure distribution in their initial crosssection: 


$$
\left.\begin{array}{rl}
\eta & =\frac{h}{h_{1}}=1+\left(\eta_{c}-1\right) \mathrm{cn}^{2}\left(\frac{x}{\Delta}, k\right), \\
\Delta & =2 h_{1} \sqrt{\frac{\eta_{c} F r_{1}}{3\left(\eta_{c}^{2}-F r_{1}\right)}}, \\
k & =\sqrt{\frac{\eta_{c}\left(\eta_{c}-1\right)}{\eta_{c}^{2}-F r_{1}}} \\
\eta_{c} & =\frac{1}{2}\left[t_{1}+F r_{1}+\sqrt{\left(t_{1}+F r_{1}\right)^{2}-4 F r_{1}}\right] .
\end{array}\right\}
$$

where $\eta_{c}=h_{c} / h_{1}$ - dimensionless depth under the first wave crest; $h_{1}, F r_{l}-$ depth and Froude number in the initial cross-section of phenomenon, respectively; $t_{1}-$ coefficient of hydrodynamic pressure; $\Delta$ and $k$ - parameters of cnoidal waves.

Studying the free-surface profile of near-critical flows allows determination of the maximum depth, the length and other parameters of the phenomena.

To check the mathematical model and to investigate the other parameters of near-critical flows, a large number of experiments of such types of near-critical flow as cnoidal waves, solitary wave and undular jump were performed in The National University of Water and Environmental Engineering [2].

A comparison between the theoretical free-surface profile was conducted, which was calculated by equation system (1) and experimental data (Fig. 2).

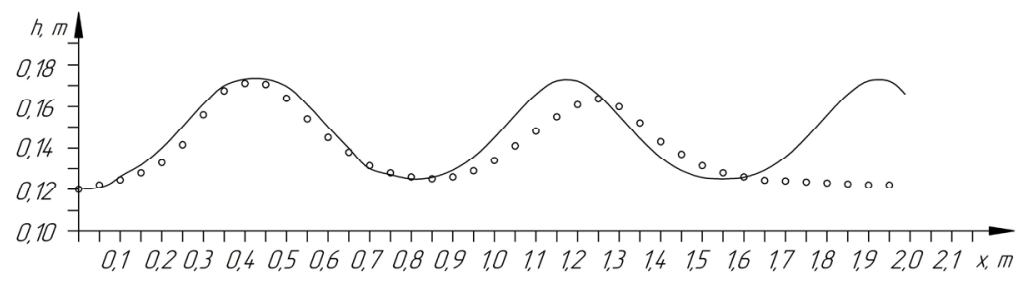

Fig. 2. Comparison of theoretical free-surface profiles of undular jump with data of laboratory research: - - theoretical profile of the free-surface of undular jump calculated by the suggested method, $\bigcirc-$ data of laboratory researches: $q=0.141 \mathrm{~m}^{2} / \mathrm{s}, h_{1}=0.12 \mathrm{~m}, F r_{1}=1.17$.

\subsection{Full-scale measurement and mathematical modelling of translation waves}

The investigation of translation waves was performed for the water reservoir of Dniester PSPP by carrying out full-scale measurement. The theoretical studying was based on "shallow water" theory and was used as a mathematical model for describing the streams of water flow in the forebay of Dniester PSPP [7]. The corresponding system of differential two-dimensional Saint-Venan equations, with the non-horizontality of the bottom and bottom friction taken into account, has the form

$$
\begin{gathered}
\frac{\partial h}{\partial t}=-\frac{\partial Q_{1}}{\partial x}-\frac{\partial Q_{2}}{\partial y}, \\
\frac{\partial Q_{1}}{\partial t}=-\frac{\partial}{\partial x}\left(\frac{Q_{1}^{2}}{h}\right)-\frac{\partial}{\partial y}\left(\frac{Q_{1} Q_{2}}{h}\right)-g h \frac{\partial(h+Z)}{\partial x}+T_{1}, \\
\frac{\partial Q_{2}}{\partial t}=-\frac{\partial}{\partial x}\left(\frac{Q_{1} Q_{2}}{h}\right)-\frac{\partial}{\partial y}\left(\frac{Q_{2}^{2}}{h}\right)-g h \frac{\partial(h+Z)}{\partial y}+T_{2},
\end{gathered}
$$


where $t$ is the time; $x$ and $y$ are spatial coordinates; $h$ is the water depth; $Q_{1}, Q_{2}$ are the specific discharges along the $x$ and $y$ axes; $Z$ is the bottom mark; $g$ is the acceleration of free fall; $T_{1}$, $T_{2}$ is the bottom friction along the $x$ and $y$ axes.

The comparison between the data of mathematical modelling and the data of full-scale measurement which was carried out by means of pressure sensors is shown in Fig. 3.

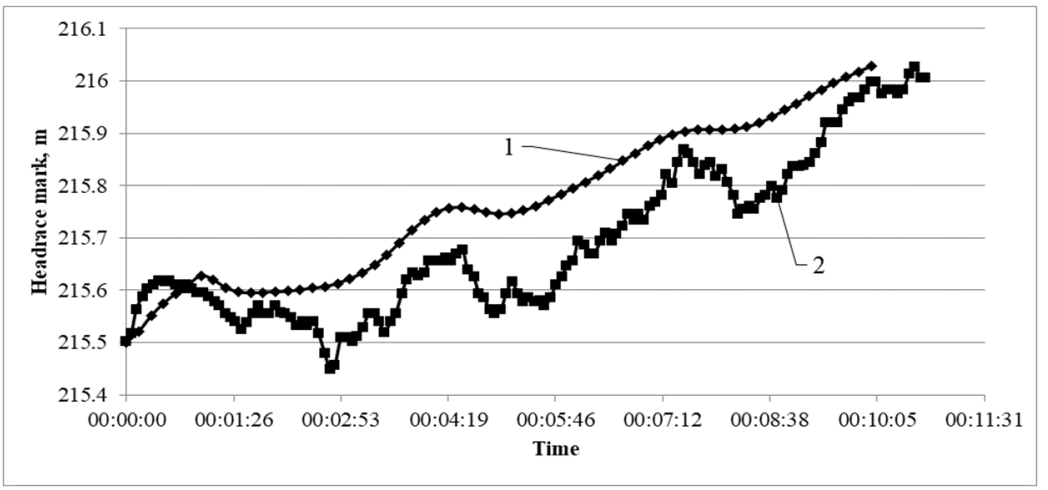

Fig. 3. Graph of comparing the results of mathematical simulation and full-scale research in the forebay of the water intake at the moment of startup of the Dniester PSPP hydraulic units in pumped mode: 1 results of mathematical simulation, 2 - results of full-scale measurement.

\section{Conclusions}

During the operation of free-flow hydraulic structures, different types of near-critical flows can occur and have negative effects on the condition of structures. Thus, the main parameters and particularities of these flows should be taken into account during the design, construction and operation of such structures.

Ignoring the aforesaid particularities can lead to false understanding of the obtained results, paradoxes and mistakes that can lead to additional costs relating to structural reconstruction or the elimination of consequences of the negative influence of near-critical flows. It is very important to use mathematical modelling, hydraulic simulation and full-scale measurement when studying the considered phenomena. The suggested mathematical models of translation waves and wave-like near-critical flows showed good convergence with experimental data.

\section{References}

1. O. A. Riabenko, O. O. Klyuha, V. S. Tymoshchuk, O. O. Halych, Lect. Notes Civ. Eng. 47, 384-391 (2020)

2. O. Riabenko, V. Chernyuk, O. Klyuha, O. Halych, D. Poplavskiy, JCEEA, 65(3/18), 121-129 (2018)

3. O. A. Riabenko, Thesis for academic degree of doctor of technical sciences, (Ukraine, 2003)

4. H. Chanson, Report CH 45/95, The University of Queensland, 202 (1995)

5. A. Giuliano, Wasserwirtschaft, 12, 501-509 (1983)

6. A. A. Tursunov, Izvestiya VNIIG, 90, 201-224 (1969)

7. O. A. Riabenko, V. S. Tymoshchuk, O. O. Kliukha, O. O. Halych, D. M. Poplavskyi, J. Mech. Eng. 22(3), 21-25 (2019) 